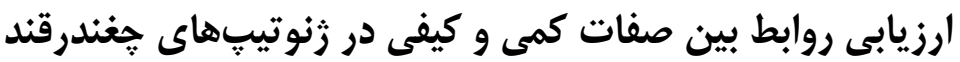 تحت شرايط آلودكى با بيمارى وكى رايزو دوكتونيا
}

\author{
اسمعيل نبىزاده' و كيوان فتوحى'
}

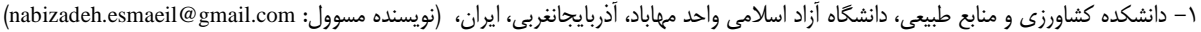

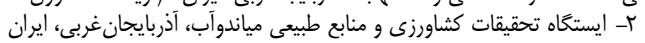

تاريخ دريافت:

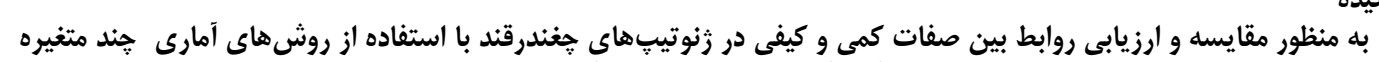

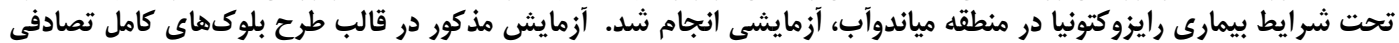

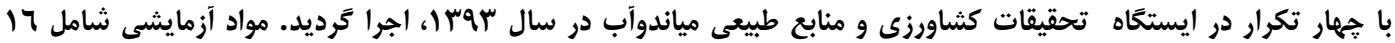

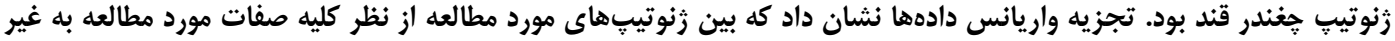

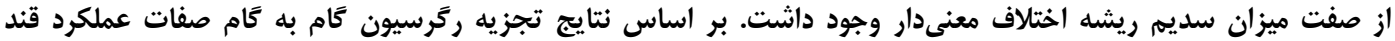

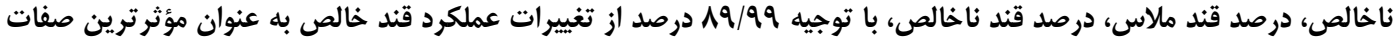

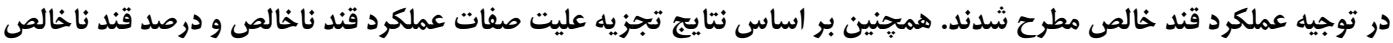

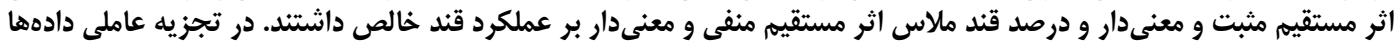

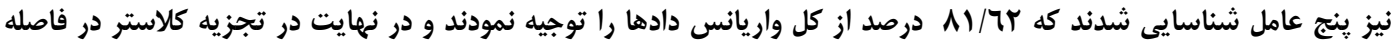

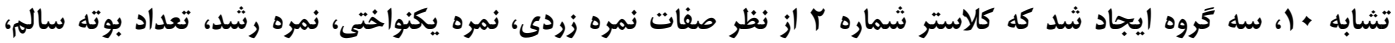

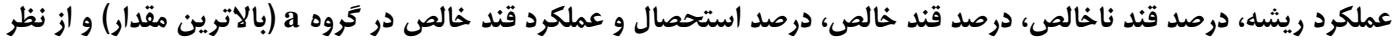

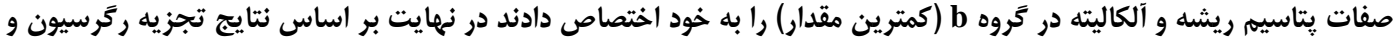

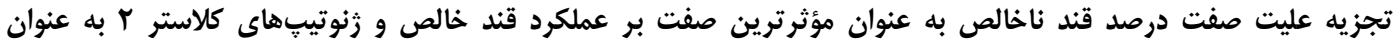

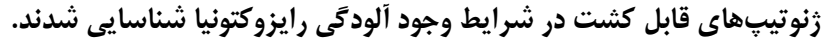

وازههاى كليدى: رايزوكتونيا، عملكرد ريشه، تجزيه به عاملها، تجزيه كلاستر

جنين بله رنغ سفيد تا قهوهاى تيره روى محيط كشت

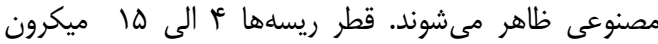

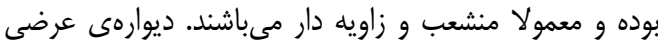

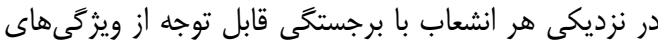

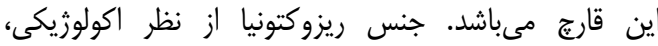

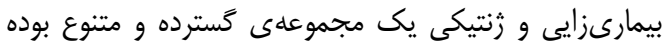

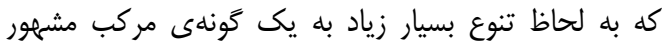

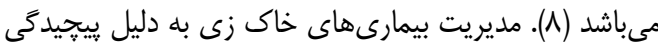

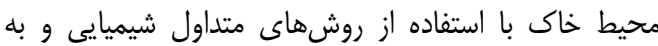

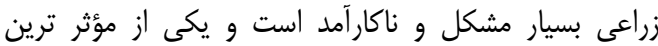

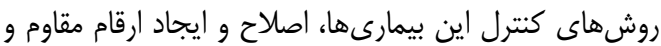

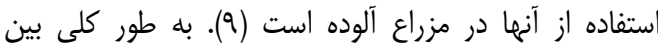

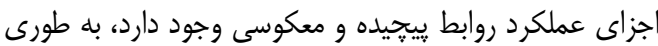

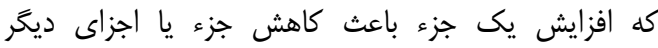

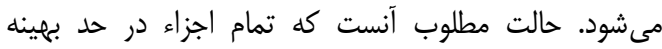

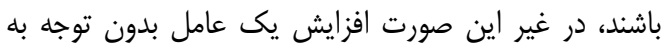

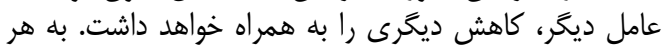
حال براى افزايش عملكرد بايستى اجزاى دائ عملكرد به به صوراه

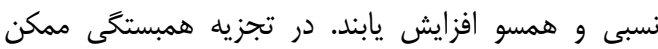

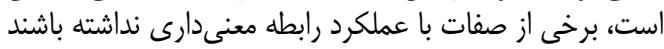

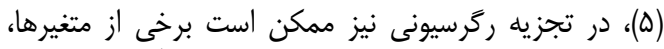

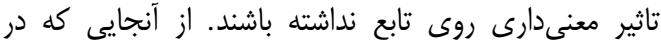

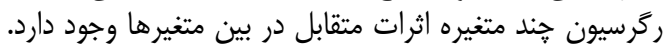

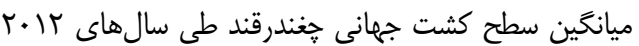

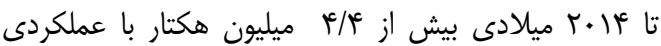

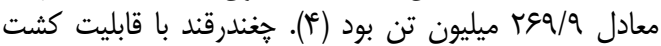

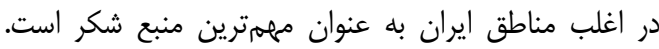

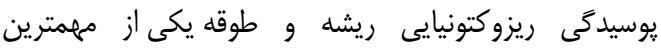

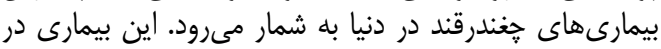

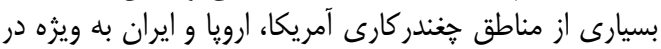

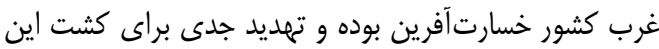

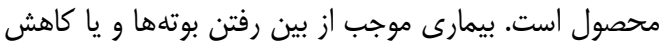

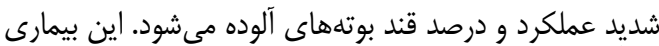

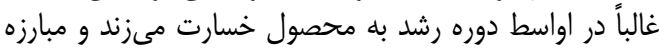

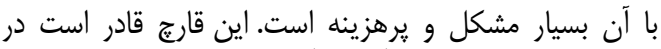

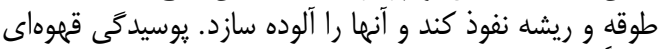

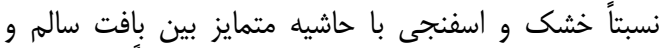

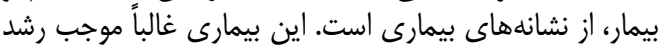

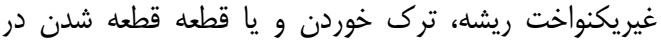

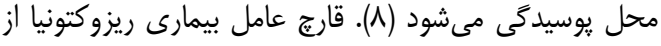

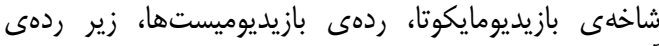

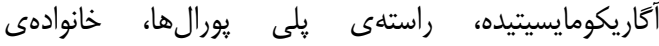

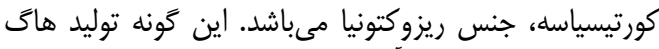

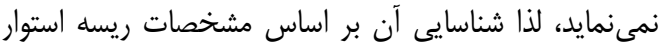

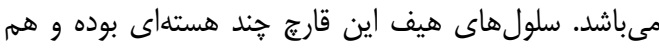




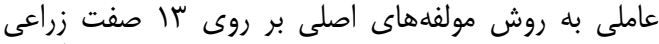

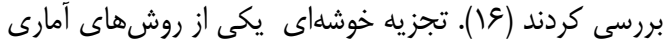

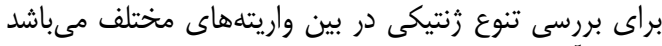

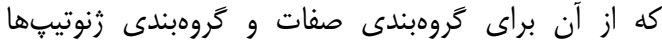

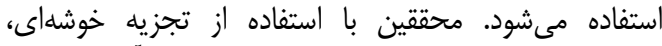

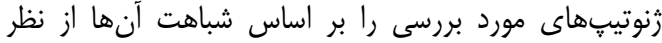

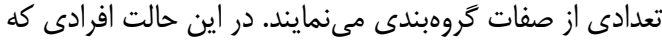

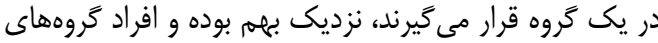

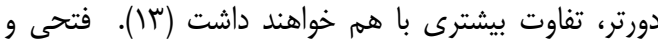

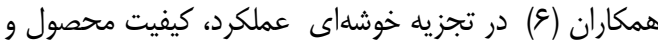

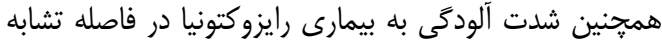

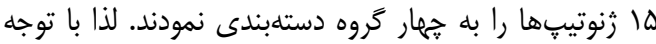

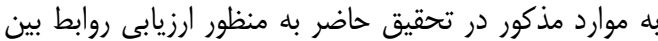

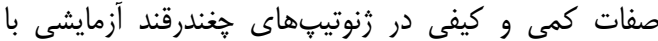

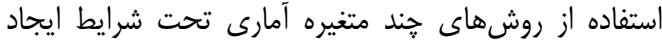
بيمارى رايزوكتونيا در منطقه مياندوآب انجام شد.

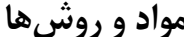

به منظور مقايسه خصوصيات كمى و كيفى ارقام داخلى و

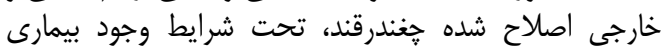

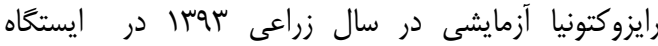

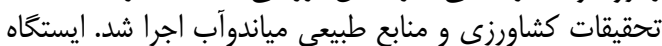

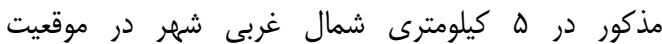

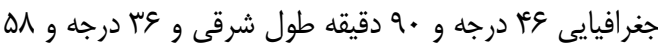

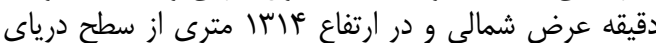

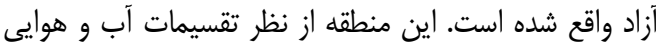

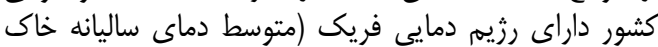

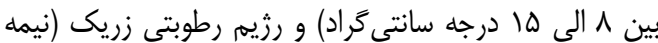

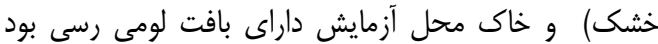

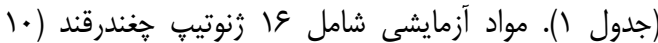

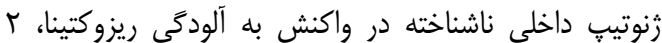

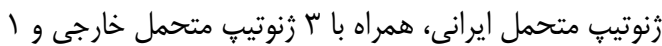

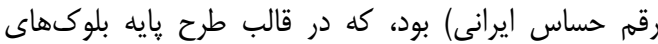

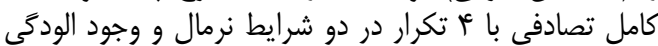

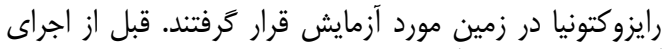

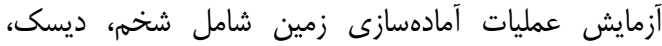

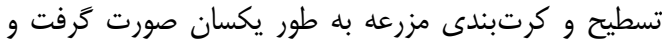

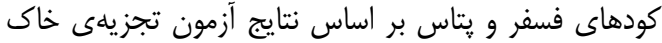

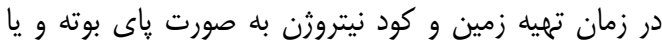

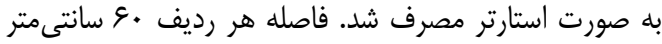

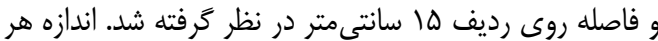

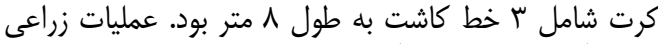

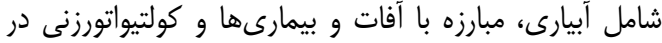

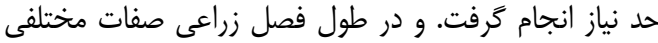

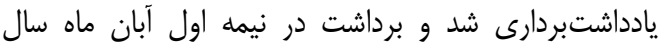

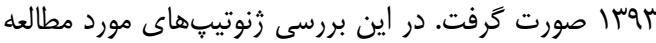

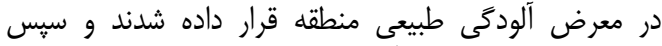

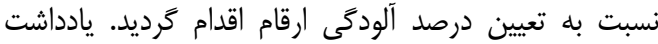
بردارى هر كرت يس از حذف نيه متر از ابتدا و انتهاى خط ئط
ممكن است يك متغير در كنار برخى از متغيرها معنى دار باشد،

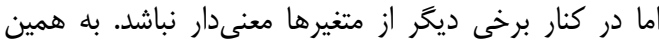

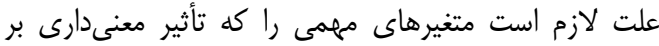

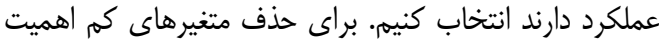

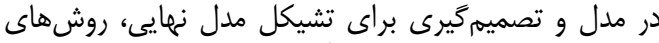

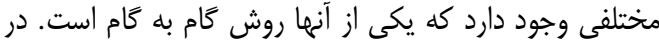

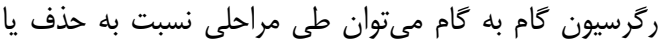

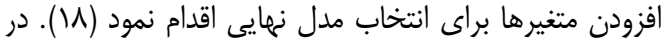

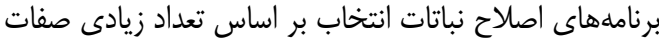

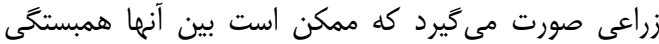

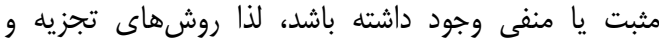

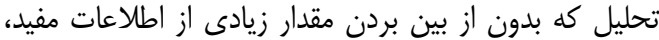

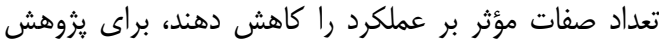

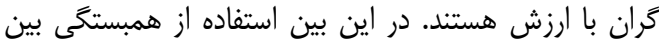

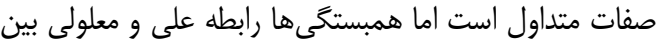

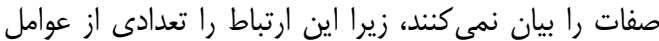

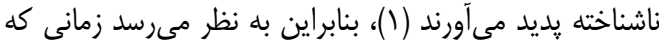

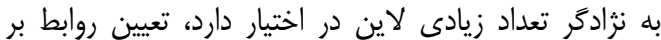

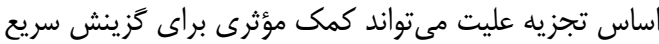

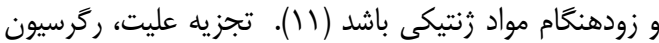

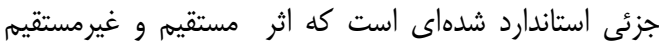

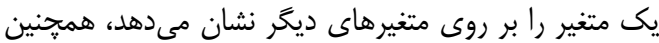

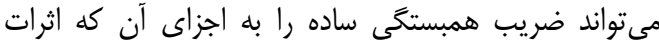

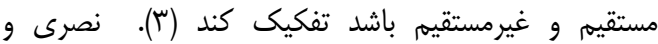

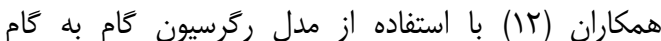

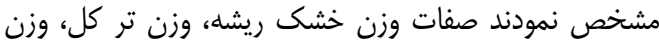

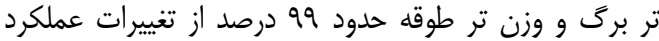

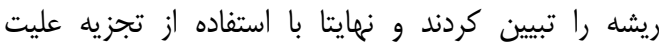

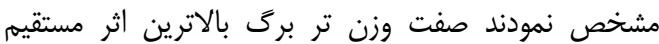

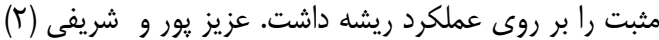

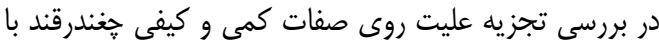

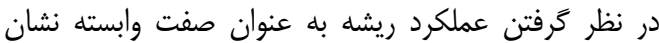

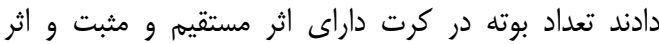

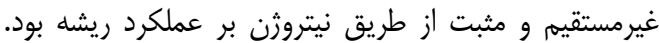

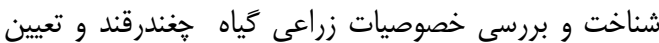

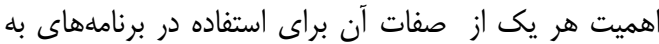

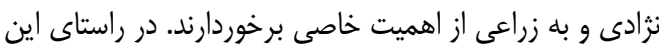

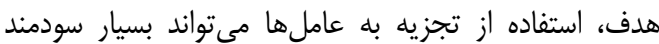

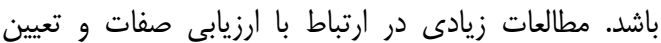

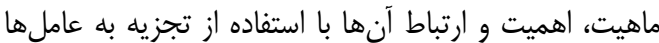

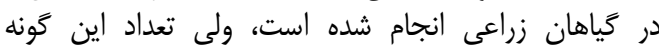

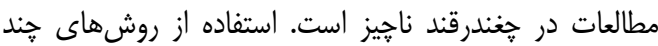

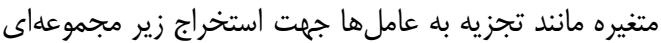

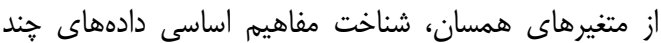

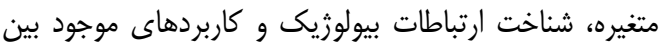

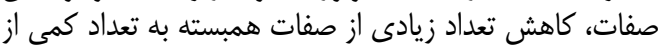

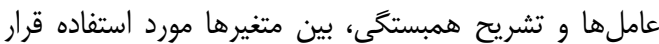

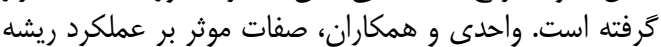
و عيارقند بر روى VD هيبريد F1 منوزرم با استفاده از تجزيه 
حذف شد. همجنين تجزيه به عاملها بر اساس تجزيه به به

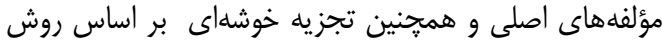

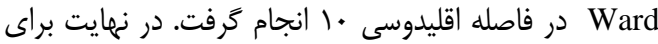
تجزيه دادهها از نرمافزارهاى آمارى Statistic a

\section{تتايج و بحث}

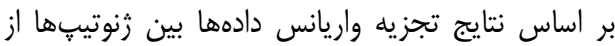

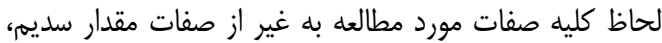

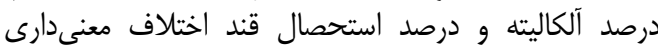

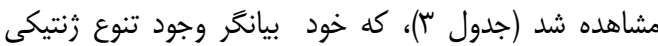
كافى بين زنوتيبها و مؤثر بودن انتخاب از لحاظ صنا صنات مورد

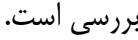

تجزيه ركر سيون و عليت بر اساس عملكرد قند خالص

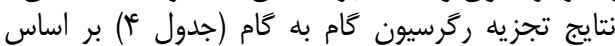

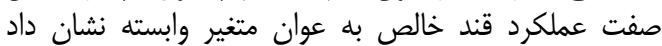

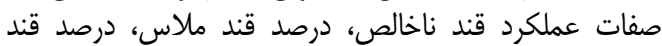

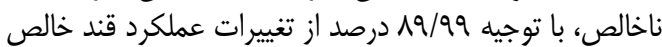

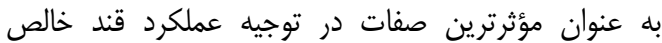

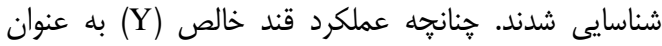

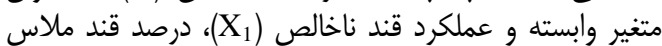
و و درصد قند ناخالص (X)

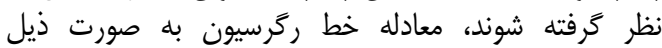

$$
\text { يردازش خواهد شد (جدول ثأ). }
$$

$Y=-\cdot / \mu T_{+} / \Delta r X_{1}-\cdot / r q X_{5}+\cdot / \cdot \Delta X_{3}$
ميانى صورت گرفت و تعداد كل بوته، بوتهاركاى سالم و و

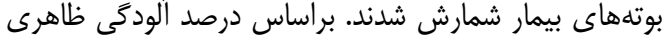

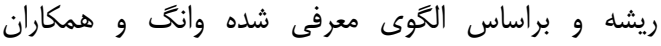

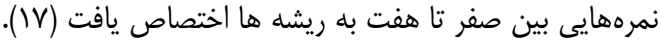

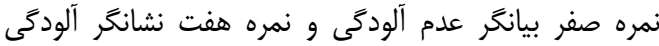

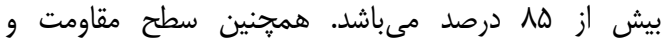

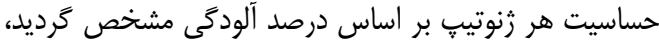

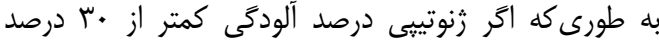

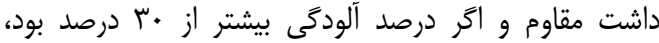

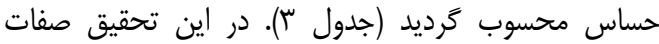

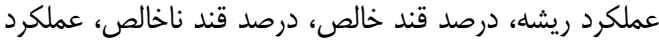

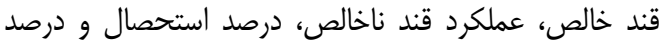

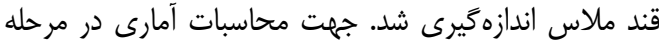

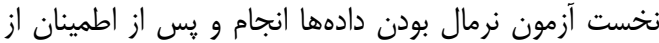

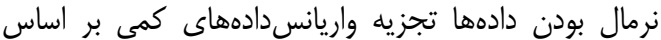

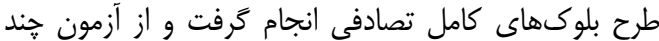
دامنهاى دانكن جهت مقايسه ميانخين ها استفاده شاند

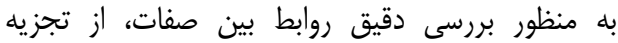
ركرسيون كام به كام و تجزيه عليه عليت استفاده شد كه در در آن آن صفت عملكرد قند ريشه به ديه دليل اهميت اقديت اقتصادى به به عنوان

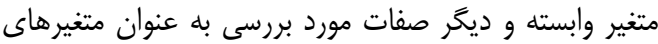
مستقل در نظر ترفتله شدند.

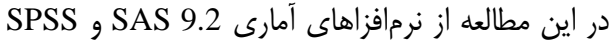

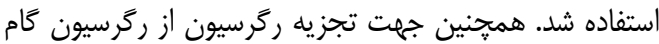

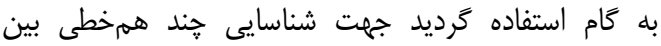

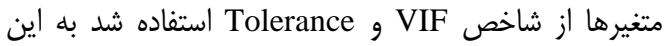

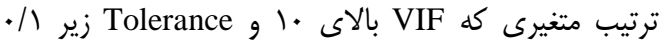
داشت به دليل شركت در حند همخطى از مدل ركرسيونى 
Table 3. Analysis of variance of experimental traits

جدول ب- تجزيه واريانس صفات مورد بررسى

\begin{tabular}{|c|c|c|c|c|c|c|c|c|c|c|c|c|c|c|}
\hline \multirow{3}{*}{ ق ق قلد ملاس } & \multicolumn{14}{|c|}{ يين مربعا } \\
\hline & عملكرد قند & درصد & عملكرد & درصد قند & آلكاليته & ازت مضره & يتاسيم & سديم & درصد قند & عملكرد ريشه & درصد آلودگىى & نمره زردى & درجه & منابع تغيير \\
\hline & خالص & استحصال & 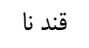 & خالص & & & ريشه & ريشه & ن اخالص & & & & آزادى & \\
\hline & & & خالص & & & & & & & & & & & \\
\hline$\cdot 1 \cdot 4^{\mathrm{ns}}$ & $1 / \wedge^{n s}$ & $\mathrm{~V} / \cdot \lambda^{\mathrm{ns}}$ & $r / 9 r^{\mathrm{ns}}$ & $.19 \Lambda^{\mathrm{ns}}$ & $\cdot / \Delta r^{\mathrm{ns}}$ & $\cdot / 1^{\mathrm{ns}}$ & $1 / 1^{\mathrm{ns}}$ & $1 / 1^{\mathrm{ns}}$ & $1 . \mid 91^{\mathrm{ns}}$ & $11 \cdot / \kappa^{4 n s}$ & $11 / 9 v^{\mathrm{ns}}$ & $\cdot / \pi \varepsilon^{\mathrm{ns}}$ & $r$ & تكرار \\
\hline . & $V / \widetilde{\sim} \Lambda^{*}$ & $\mid \varepsilon / v \varepsilon^{\mathrm{ns}}$ & $1 . / 1 \mu^{*}$ & $\Delta / \Gamma^{*}: *: *$ & $s / q^{\mathrm{ns}}$ &.$/ \Gamma \Delta^{*}$ & $r / \cdot r^{*}$ & $r / \Delta)^{\mathrm{ns}}$ & $\Delta \cdot / 9 \cdot * *$ & $19 \cdot / V^{* * *}$ & MMN/rq"**: & $1 / \Delta \Lambda^{* * *}$ & 10 & رُنوتيب \\
\hline .1 .9 & 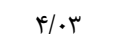 & $11 / r v$ & $\Delta / 8 \Delta$ &.$/ 99$ & $1 / 99$ &.$/ 1 \Lambda$ & $1 / \cdot r$ & $r / T r$ & $\mid \omega / \& \Delta$ & $119 / \pi 9$ & $|c / 4|$ & . & is & خطا آزمايشى \\
\hline $\mid r / 1$ & tr/AT & $r / I T$ & $r T / g)$ & V/Ar & $19 / 94$ & ( & $1 F / \Delta q$ & $I T / V T$ & $r V / \Delta q$ & rQ/qr & $r \cdot / 9 \Delta$ & $\mid Q / V \wedge$ & - & ضريب تغييرات CV\% \\
\hline
\end{tabular}

جدول عأ- مراحل ركرسيون كام به كام براى عملكرد قند خالص به عنوان متغير وابسته و ساير متغيرها به عنوان مستقل

Table 4. Stepwise regression of pure sugar yield as dependent variant and others as independent

\begin{tabular}{ccc}
\hline & \\
\hline & & \\
\hline \\
\hline
\end{tabular}




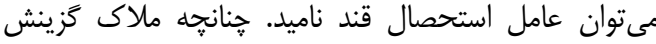

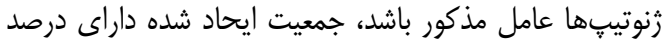

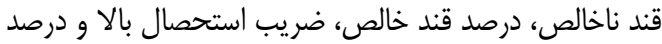

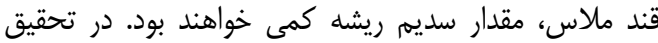

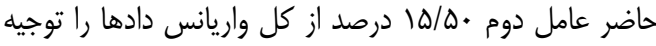

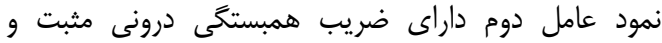

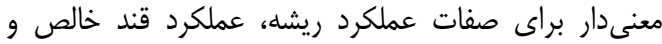

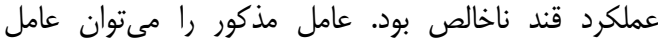

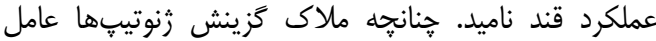

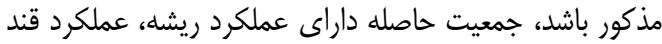

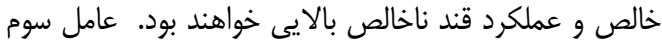

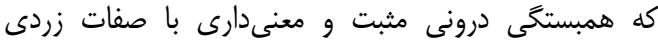

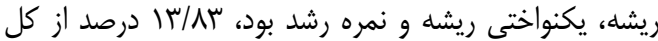

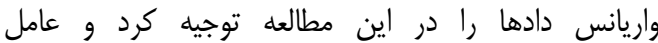

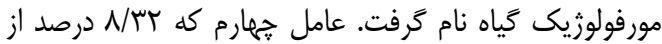

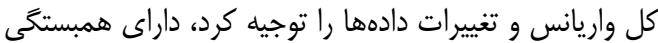
درونى مثبت و معنى معنى دارى با صفت دادها تعداد

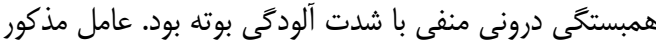

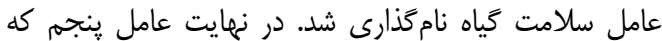

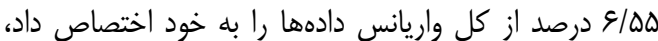
داراى ارتباط درونى مثبت با دران صفات ازت داده مضره، آلكاليته،

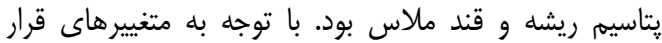

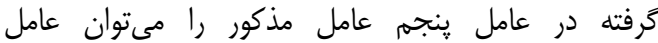

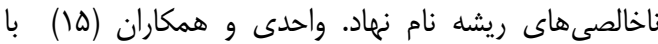

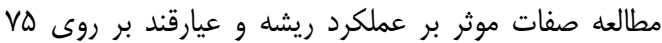

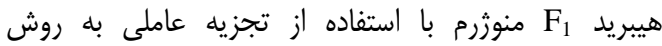

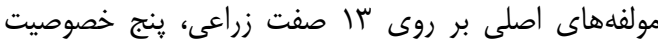

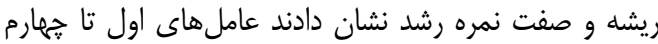
به ترتيب ه\&/F

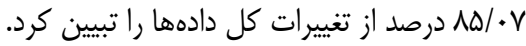

تجزيه عليت (جدول ه) بر اساس صفات باقى مانده در

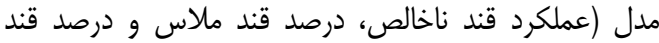

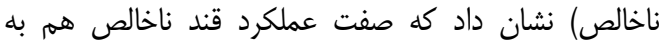

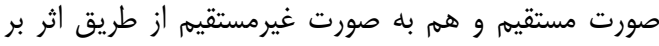

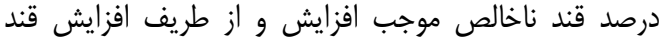

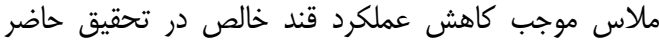

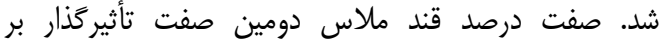

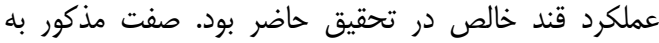

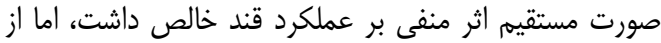

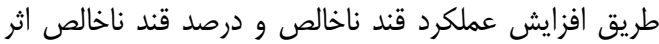

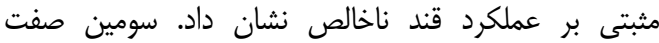

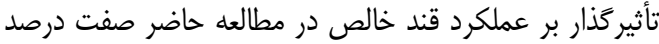

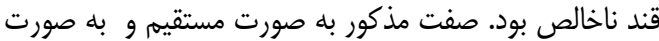

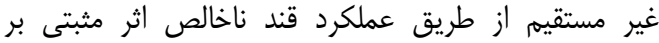

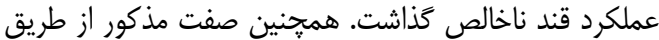

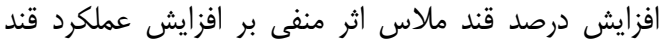

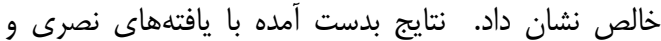

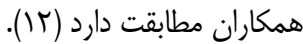

تجزيه به عاملها مطان دار

در تحقيق حاضر مقدار

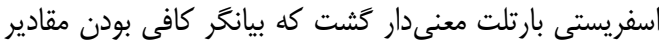

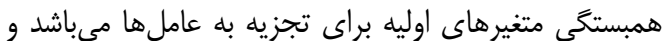

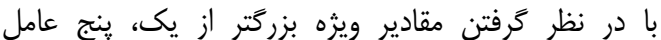

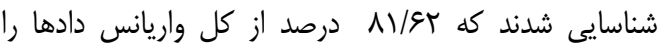

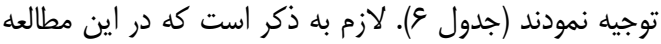

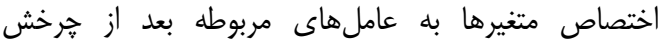

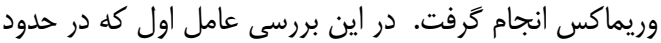

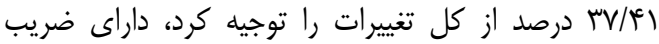

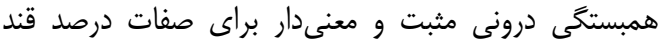

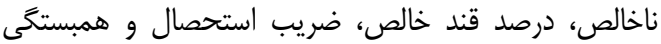

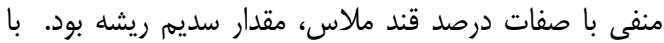

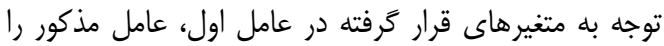

Table 5. Indirect and direct effects of the traits on the pure sugar yield

جدول ه- اثر مستقيم و غير مستقيم صفات بر عملكرد قند خالص

\begin{tabular}{cccccc}
\hline & & & \\
\hline \\
\hline
\end{tabular}


Table 6. Factor analysis for the traits after Varimax rotation

$$
\text { جدول צ- تجزيه به عاملها صفات بعد از جرخش وريماكس }
$$

\begin{tabular}{|c|c|c|c|c|c|}
\hline \multicolumn{6}{|c|}{ بار عامل ها } \\
\hline ينجم & جهارم & سوم سوم & دوم & اول اول & صفات \\
\hline$\cdot / 11$ & $\cdot / T V$ &.$/ A r$ &.$/ \cdot \Delta$ & .110 & زردى \\
\hline .1 .4 & $\cdot /$ & $\overline{. / M}$ & $\cdot / M F$ &.$/ \Lambda$ & يكنواختى ريشه \\
\hline$\cdot / \cdot v$ & $\cdot / M F$ &.$/ 1 \wedge \Delta$ &.$/ 10$ &.$/ 19$ & نمره رشد \\
\hline$\cdot / \cdot v$ &.$/ V 9$ & $\overline{. / \mu 1}$ & 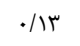 &.$/ T \Lambda$ & تعداد بوته سالم \\
\hline$-\cdot / \cdot V$ &.$- / 19$ &.$- / 1 \mathrm{~V}$ &.$- / 1$ & $.1 . e^{c}$ & شدت آلودگى \\
\hline.$/ . r$ & $\overline{.1 .0}$ &.$/ 14$ & .198 & $\cdot / V$ & عملكرد ريشه \\
\hline.$/ .0$ & .1 .9 & 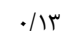 & .191 &.$/ 19$ & عملكرد قند ناخالص \\
\hline.$/ . r$ &.$/ \cdot v$ &.$/ r$ & .190 &.$/ T \Delta$ & عملكرد قند خالص \\
\hline.$/ 19$ & .119 & .1 .9 & $\overline{. / 1 \pi}$ & .119 & درصد قند نا خالص \\
\hline.$/ V^{c}$ & 每 & 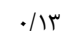 &.$/ 1$ &.$- / 0$ & قند ملاس \\
\hline$\overline{. / 14}$ & $\cdot / 19$ & $-* / 1 \wedge$ & 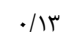 &.$/ V^{f}$ & درصد قند خالص \\
\hline.$/ \mathrm{V} 9$ &.$- / 19$ &.$/ \pi$ &.$/ \cdot v$ & $\overline{.1 .4}$ & ازت مضره \\
\hline .199 &.$- / 48$ & 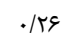 & 每- &.$- / r r$ & آلكاليته \\
\hline$\overline{.1 \Delta \Lambda}$ &.$- / 199$ & $-* / 1 \Lambda$ & .1 .9 &.$- / 4 V$ & يتاسيم ريشه \\
\hline$\overline{. / 19}$ & $\cdot / M F$ &.$/ 14$ & 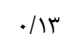 & .119 & ضريب استحصال \\
\hline . & .11 & .119 &.$/ 14$ &.$- / \mathrm{NT}$ & سديم ريشه \\
\hline $1 / . F$ & וr & $T / r)$ & $t / F \Lambda$ & $\Delta / 9 \Lambda$ & ريشه مشخصه \\
\hline$\varepsilon / \Delta \Delta$ & $N / H r$ & I & $1 \phi / \Delta$ & $r V / s^{e}$ & نسبت واريانس توجيه شده \\
\hline NN/AT & $v \Delta / \cdot v$ & $g g / V^{4}$ & $\Delta r / 91$ & $r V / s i$ & جمع كل واريانسهاى توجيه شده \\
\hline
\end{tabular}

F- 31873، 31868، S-20654 20655، 31079، 31870،

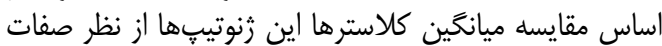

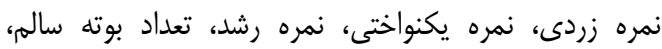

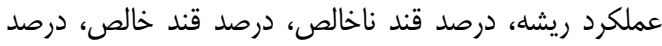

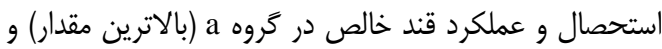

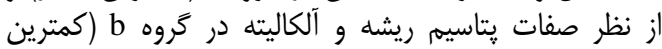

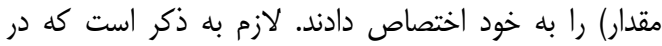

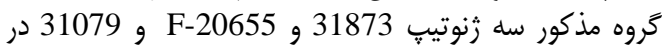

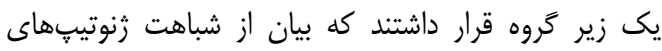

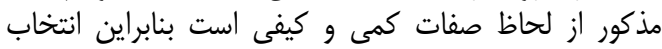

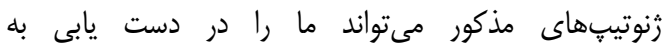

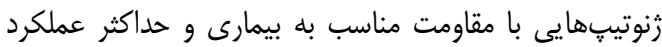

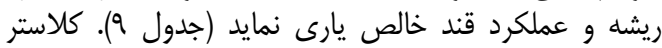

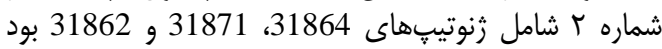

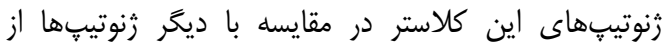

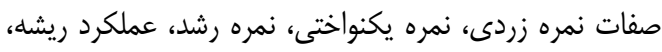

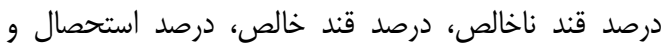

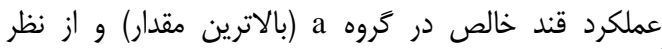

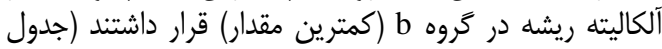

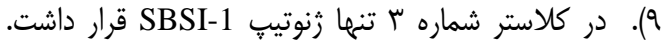

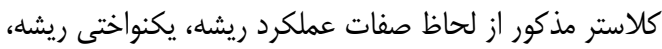

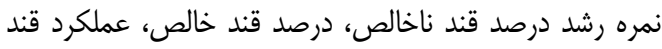

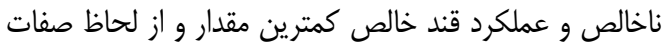

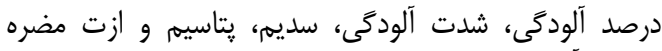

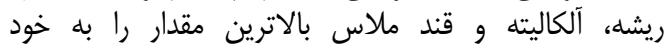

\section{تجزيه خوشهاى زنوتينها}

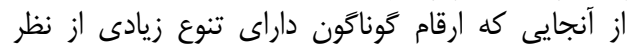

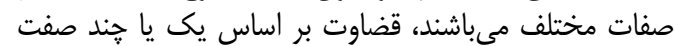

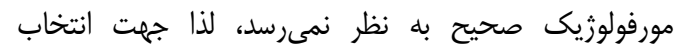

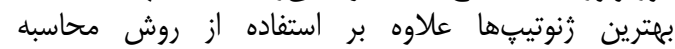

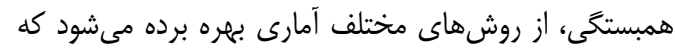

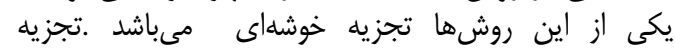

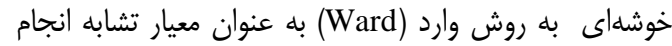

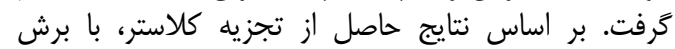

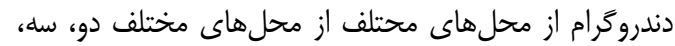

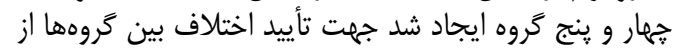

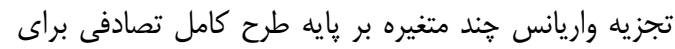

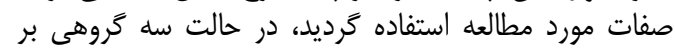

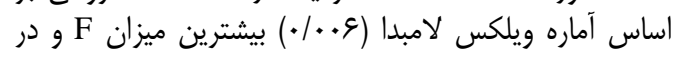

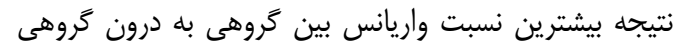

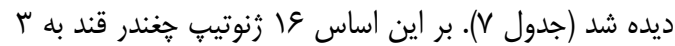

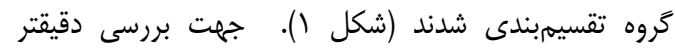

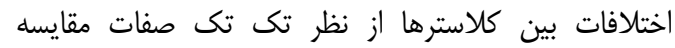

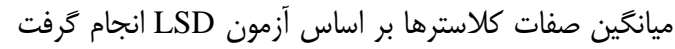

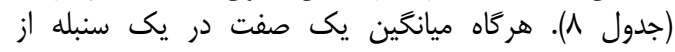

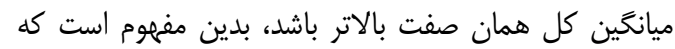

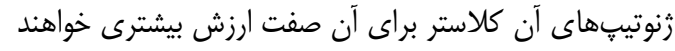

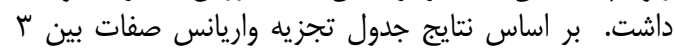

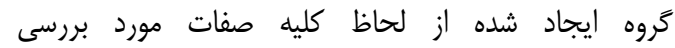

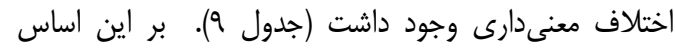

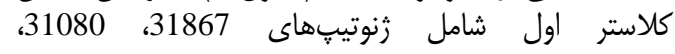


Polyrow

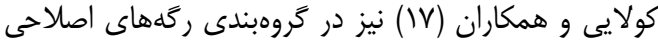

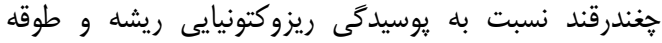

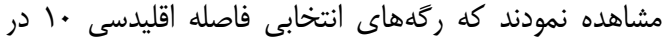

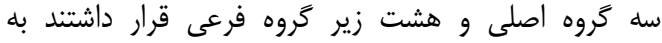

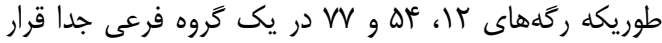

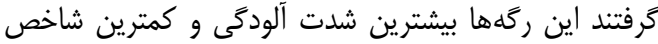

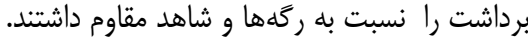

اختصاص داد و به عنوان نامناسبترين زنوتيٍٍ شناسايى شد

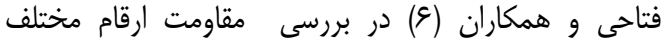

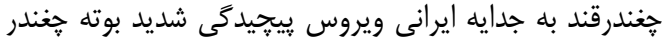

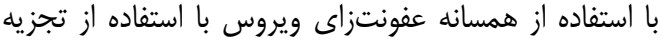

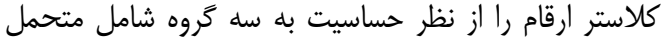

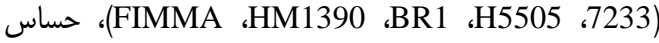
(رسول، افشارى، هيبريد بالك شيراز، زرقان، Hilma، Flores g P.P.8 ،P.P.22 (Dorothea ،Rhizofouret ،IC

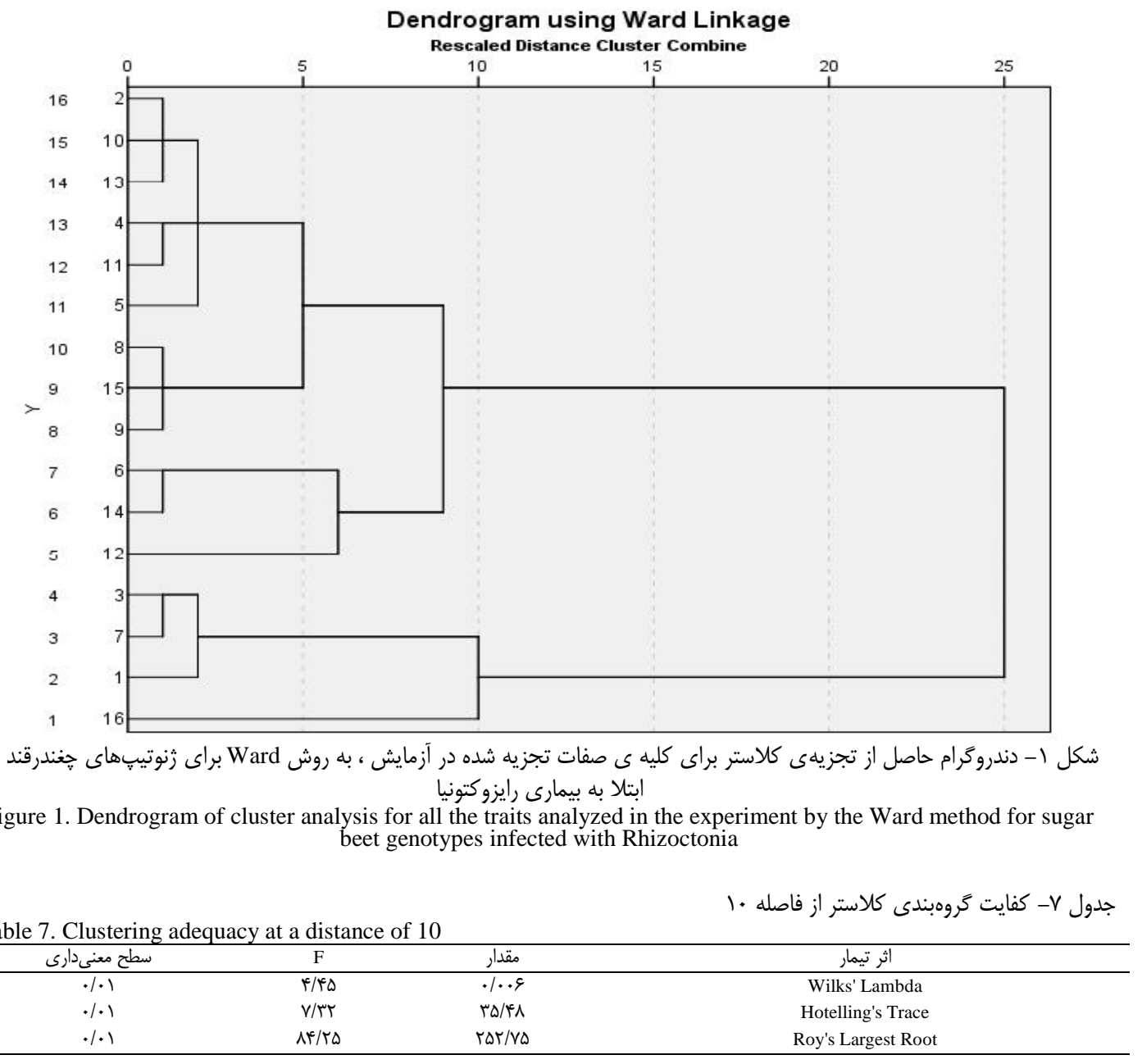

جدول ^- تجزيه واريانس بين و درون كلاسترهاى مورد بررسى در فاصله •10 1. Table 8. Analysis of variance in and between the studied clusters at a distance of 10

\begin{tabular}{|c|c|c|c|c|c|c|c|c|c|}
\hline \multicolumn{8}{|c|}{ ميانگين مربعات } & \multirow{3}{*}{ آزاديى } & \multirow{3}{*}{ منابع تغيير } \\
\hline درصد قند & عملكرد & ضريب & لدرصد & تعداد بوته & نمره & نمره & نمره زردى & & \\
\hline ناخالص & ريشه & ألودَى & ألودگى & سالم & رشد & يكنواختى & & & \\
\hline$\Gamma / \Delta \omega^{* * *}$ & $194 / \backslash \varphi^{*}$ & $r l l / \Delta \gamma^{* *}$ & 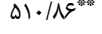 & $1 \Delta \omega / ৭ \Lambda^{* *}$ & $1 / 99^{* 3}$ & $1 / \mathrm{rq}^{9 * 4 / 4}$ & $1 / 4 \gamma^{*}$ & r & بين كروهها \\
\hline .190 & $99 / \mathrm{Vr}$ & IS/QT & $I T / K r$ & $T r / F G$ &.$/ 4 \pi$ & $.1 / 9$ & $\cdot / 4 \pi$ & 10 & درون گروه \\
\hline
\end{tabular}



ارزيابى روابط بين صفات كمى و كيفى در زنوتيڤهاى خغندرقند

Continued Table 8

ادامه جدول ^- تجزيه واريانس بين و درون كلاسترهاى مورد بررسى در فاصله .1

\begin{tabular}{|c|c|c|c|c|c|c|c|c|c|}
\hline & & & & & مربعات & & & & \\
\hline خالصرد قند & درمد قند & استحصال & خالص قرند & آلكاليته & ازت مضره & يتاسيم & سديم ريشه & درجه أزادى & منابع تغيير \\
\hline $\mathrm{F} / \mathrm{V} \mathrm{V}^{*}$ & $\cdot / \cdot 1^{\mathrm{ns}}$ & $|\varepsilon / 1|^{*}$ & $r / 9 \Lambda^{* * * 3}$ & 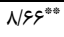 & $\cdot / \cdot^{\mathrm{ns}}$ & $r / A r^{\text {s.* }}$ & $\cdot / r \cdot{ }^{n s}$ & $r$ & بين كروه ها \\
\hline $1 / \% q$ & .1 .9 & $T / T_{\Delta}$ &.$/ 94$ & .109 & $.1+1$ &.$/ 44$ &.$/ T \Delta$ & 10 & درون گروه \\
\hline
\end{tabular}

جدول q- مقايسه ميانخين صفات كلاسترها و ميانخين كل در صفات مورد ارزيابى Table 9. Means comparison of the cluster traits and total mean of the experimental traits

\begin{tabular}{|c|c|c|c|c|c|c|c|}
\hline درصد قند ناخالص & عملكرد ريشه & درصد آلودگى & تعداد بوته سالم & رشده & نمره يكنواختى & نمرهزردى & كلاستر \\
\hline $1 \omega / V r^{a}$ & $r \varepsilon / \mathrm{VV}^{\mathrm{a}}$ & $I T / I e^{b}$ & $r V / V r^{a}$ & $r / \Delta f^{a}$ & $r / F \gamma^{a}$ & $r / F \Delta^{a}$ & كلاستر 1 \\
\hline $\mid Q / q^{a}$ & $f \cdot / \mu \mu^{a}$ & $r N \cdot l^{a}$ & $m / / q^{a b}$ & $\mathrm{r}^{\mathrm{a}}$ & $r / r \Delta^{a}$ & $\mathrm{r}^{\mathrm{a}}$ & كلاستر r \\
\hline $\mid r / a \varepsilon^{b}$ & $M^{b}$ & $r \varepsilon / f)^{a}$ & $M M / \Delta \cdot{ }^{b}$ & $1 / V \Delta^{b}$ & $1 / V \Delta^{b}$ & $1 / V \Delta^{b}$ & كلاستر r \\
\hline
\end{tabular}

\begin{tabular}{|c|c|c|c|c|c|c|c|c|}
\hline \multicolumn{3}{|c|}{ Continued Table 9.} & \multicolumn{3}{|c|}{ و ميانخين كل در صفات مورد ارزيابى } & \multicolumn{2}{|c|}{ قايسه ميانخين صفات كلاسترها } & جدول 9- \\
\hline خالصرد قند & مراس قند & درصد استحصال & درصد قند خالص & آلكاليته & ازت مضره & يتاسيهم ريشه & سديم ريشه & كلاستر \\
\hline$f / V v^{a}$ & $T / / F^{a}$ & a & $1 r / 99^{a}$ & $g / r^{b}$ & $1 / 99^{a}$ & $\varepsilon / \wedge \gamma^{b}$ & $\varphi / \cdot \Delta^{\mathrm{a}}$ & كلاستر 1 \\
\hline$\Delta / T F^{a}$ & $r / r \Delta^{\mathrm{a}}$ & $\Lambda \mathrm{M} / \mathrm{V}^{\mathrm{c}^{\mathrm{a}}}$ & $\mid r / q V^{\mathrm{a}}$ & $g / 8 \Lambda^{b}$ & $1 / V^{a}$ & $N / F^{a^{a}}$ & $r / \leftarrow \varepsilon^{a}$ & كلاستر r \\
\hline $1 / v \xi^{b}$ & $r / \Lambda \Lambda^{a}$ & $V \varepsilon / q^{b}$ & $1 . / 1 v^{b}$ & $1 . / 8 r^{\mathrm{a}}$ & $1 / r \cdot{ }^{\mathrm{a}}$ & $V / \wedge q^{a b}$ & $r / q^{a}$ & كلاستر r \\
\hline
\end{tabular}

منابع

1. Acquah, G., N.W. Adams and J.O. Kelly. 1992. Factor analysis of plant variable associated with architecture and seed size in day bean. Euphtica, 60: 171-177.

2. Aziz Poor, M. and H. Sharifi. 2008. Path analysis on quantitative and qualitative characteristics of Scabies, Warts Chghnzr Sugar. Tehran.Pardis Aburaihan University $.10^{\text {th }}$ Agronomy and Plant Breeding Abstracts.1364.pp (In Persian). https://www.civilica.com/Paper-NABATAT10-NABATAT10_285.html

3. Dewey, D.R. and R.H. Lu. 1959. A correlation and path coefficient analysis of components of crested wheat grass seed production. Agronomy Journal, 51: 515-518.

4. FAO (Food and Agriculture Organization) Rome. 2014.http//faostat.fao.org/.

5. Farshadfar, A. 2000. Principles and multivariate statistical methods. Razi University of Kermanshah, 754 pp (In Persian).

6. Fathi, M.R., S.A. Vahedi, M. Bazrafshan, H. Shahbazi and C.E. Abdollahyan Noghabi. 2012. Preparation of hybrids of sugar beet rhizomania disease resistance gene and comparison of performance and quality. Seed and Plant Journal, 29(4): 777-789.

7. Fattahi, Sh., D.M. Zafari and S.B. Mahmoudi. 2011. Evaluation of superior genotypes of sugar beet resistance to the important root rot agents under greenhouse conditions. Journal of Sugar Beet, 27(1): 2538 (In Persian).

8. Harveson, R. 2002. Rhizoctonia Root and Crown Rot of Sugar Beet. The Board of Regents of the University of Nebraska on behalf of the University of Nebraska-Lincoln Extension.

9. Hecker, R.J. and E.G. Ruppel. 1975. Inheritance of resistance to Rhizoctonia root rot in sugar beet. Crop Science, 15: 487-490.

10. Kolaee, H., S.B. Mahmoudi and M. Hasani. 2010. Evaluation of resistance of beet breeding lines to Rhizoctonia root and crown rot. Journal of Sugar Beet, 26(1): 31-42 (In Persian).

11. Mobaser, S. and S. Shah Moradi. 1996. Correlation between protein content of the grain yield and some agronomic and morphological traits using path analysis. Seed and Plant, 12(2): 24-29.

12. Nasri, R., A.S. Kashani, F. Paknejad, M. Sadeghiand S. Ghorbani. 2012. Correlation and path analysis of yield and quality of sugar beet in both direct seeding and transplanting of saline lands. Agriculture and Horticulture, 8(1): 226-313.

13. Romesborg, H.C. 1990. Cluster analysis for researches, R.K. Publishing Company, Malabar, Florida, 925 .

14. Sadeghian, S.Y., H. Fazli, D.F. Taleghani and M. Mesbah. 2000. Genetic variation of drought stress in sugarbeet. Journal of Sugar Beet Research, 37: 55-77.

15. Vahedi, S.M., R. Mesbah Amiri, M.R. Bihamta, V. Yusefabadi and M. Dehghanshoar. 2006. Relationship between agronomic traits and root morphological characteristics and determine traits affecting root yield and sugar content in germplasm of sugar beet monogerm. Journal of Sugar Beet, 22(2): 19-34 (In Persian).

16. Vahedi, S., D. Misbah, R. Amiri, M.R. Bihamta, V. Yusef Abad and M. Dhqanshar. 2007. Relationship between root morphological traits of agronomic traits and characteristics affecting the determination of root yield and sugar content of sugar beet Germplasm, monogerm. Sugar Magazine, 22(2): 34-19, 12(2): 24-29.

17. Wang, Y., Y. Lin, P. He, L. Chen, L. Amicarna and J.D. Lu. 1995. Evaluation of foliar resistance to Uncinula necator in Chinese Wild Vitis Species. Vitis, 34: 159-164.

18. Zinali, H., E. Naser-Abadi, H. Hossein-zadeh, R. Chugan and M. Sabokdast. 2004. Factor analysis on hybrid of cultivar grain maize. Iranian, Journal of Agriculture Science, 36(4): 895-902 (In Persian). 


\title{
Study of Relationships among Qualitative and Quantitative Traits in Sugar Beet Genotypes Infected with Rhizoctonia
}

\author{
Esmail Nabizadeh $^{1}$ and Kaywan Fotohi ${ }^{2}$
}

1- Department of Agronomy, College of Agriculture, Mahabad Branch, Islamic Azad University, Mahabad, Iran (Corresponding author: Nabizadeh.esmaeil@gmail.com)

2- Miandoab Agricultural and Natural Resources Research Station, West Azerbaijan, Iran $\begin{array}{ll}\text { Received: November 6, } 2016 & \text { Accepted: October 2, } 2017\end{array}$

\begin{abstract}
In order to study and compare the correlation between the quantitative and qualitative traits of sugar beet genotypes, using multivariate methods under Rhizoctonia disease a trial was carried out at Miandoab Agricultural and Natural Resources Station in 2014.The experimental design was randomized complete block design with four replications. The experimental materials were sixteen sugar beet genotypes. Analysis of variance showed significant differences among the genotypes for all traits, except root sodium content. Based on stepwise regression analysis, gross sugar yield, molasses sugar content and gross sugar content by justifying \%89.99 of changes in white sugar yield were considered as the most effective traits in justification of white sugar yield. Factor analysis identified five factors by justifying $81.62 \%$ of total data variances. Finally, the three groups were set up in the cluster analysis at similarity distance of 10. The cluster 2 was subjected to groups a (the highest rate) for yellowing score, uniformity score, growth score, healthy plant number, root yield, gross sugar yield, white sugar content, white sugar yield and extract percentage, and was placed in group b (the lowest rate) for root potassium content and alkalinity. Eventually, based on path analysis and regression analysis, the gross sugar yield was determined as the most effective trait on the white sugar yield and the genotypes in cluster 2 were identified suitable for cultivation under the conditions of Rhizoctonia infestation.
\end{abstract}

Keywords: Rhizoctonia, Root Yield, Factor Analysis, Cluster Analysis 